\title{
Precise predictive and therapeutic strategy for breast cancer
}

\author{
loannis D Kyrochristos ${ }^{1,2}$, Dimitra V Peristeri2 ${ }^{2}$, Demosthenes E Ziogas ${ }^{1,3}$, Efstathios G \\ Lykoudis ${ }^{4}$ \& Dimitrios H Roukos*,2,5 \\ ${ }^{1}$ Center for Biosystems \& Genome Network Medicine, Ioannina University, loannina, 45110, Greece \\ ${ }^{2}$ Department of Surgery, loannina University Hospital, loannina, 45500, Greece \\ ${ }^{3}$ Department of Surgery, 'G Hatzikosta' General Hospital, Ioannina, 45001, Greece \\ ${ }^{4}$ Department of Plastic Surgery, loannina University Hospital, loannina, 45500, Greece \\ ${ }^{5}$ Department of Systems Biology, Biomedical Research Foundation of the Academy of Athens (BRFAA), Athens, 11527, Greece \\ *Author for correspondence: Tel.: +30 265100 5572; droukos@uoi.gr
}

\section{'Gan evidence-based strategy is essential to validate promising breast cancer genome analysis in time and space."}

First draft submitted: 5 April 2018; Accepted for publication: 30 April 2018; Published online: 30 May 2018

Keywords: breast cancer $\bullet$ druggable mutations $\bullet$ drugs $\bullet$ genome analysis $\bullet$ personalized treatment $\bullet$ precise prediction • therapeutic response

Several new targeted drugs have recently been approved for the treatment of breast cancer [1]. Despite continuing progress in research and clinical practice, therapeutic resistance and relapse rates remain high in advanced disease [2]. We summarize the latest developments in genome analysis and translational therapeutic implications.

Progress in basic translational and clinical research for breast cancer has been impressive. No other cancer type has seen such rapid advancements, such as the primary prevention of hereditary breast and ovarian cancer syndrome, screening for early-stage detection and computational algorithms for decision-making on multimodal treatment [3]. Personalized prophylactic bilateral mastectomy with or without bilateral salpingo-oophorectomy is based on family history, BRCA1 or BRCA2 status and age [4]. Established mastographic screening has increased the rates of in situ and stage I disease diagnosis, substantially improving overall survival [2]. At the same time, new systemic therapeutic modalities have been developed, including chemotherapy and targeted therapy as standard treatment, as well as immunotherapy as a promising perspective.

Despite these advances, individualization of therapeutic strategies remains challenging. Currently, no robust prognostic and predictive biomarkers have been developed for exact, personalized prediction of oncological outcomes and therapeutic response. Moreover, recent evidence on extensive genetic and genomic heterogeneity [5] suggests the urgent need for the development of multiple targeted drugs to increase the rates of therapeutic response [6,7].

\section{Advances in prevention \& treatment}

Both preventive and therapeutic strategies have featured important advances. Integration of somatic and inherited mutations into clinical practice for sporadic and hereditary cancer has improved oncological outcomes and overall survival.

In the prevention setting and beyond $B R C A 1 / B R C A 2$ testing, multigene panels have been developed including numerous established cancer driver genes, involved in familial breast cancer. Distinguishing all these genes into high-, moderate- and low-penetrance, women with familial predisposition for breast cancer can be categorized into high-, moderate- and low-risk groups. This separation defines the preventive strategy for the high-risk group (BRCA1/2 mutation carriers) [4], including prophylactic surgery, such as bilateral mastectomy and/or bilateral salpingo-oophorectomy, intensive screening implementing breast MRI for moderate-risk individuals (family history and BRCA1/2 negative, moderate-penetrance genes) and traditional mammographic screening for low-risk women (low-penetrance genes) [1]. 
Two new drugs have recently been approved for the treatment of HER2-positive breast cancer [8,9]. Following advancements for HER2-positive breast cancer, including the approval of trastuzumab in 2001 for the metastatic setting and 2005 for early breast cancer, as well as trastuzumab-emtasine conjugate for metastatic cancer in 2013, pertuzumab and neratinib maleate have further improved oncological outcomes and survival. More specifically, women with HER2-positive breast cancer, in the adjuvant or neoadjuvant setting, can be treated with trastuzumab added to chemotherapy or pertuzumab $[9,10]$ in conjunction with trastuzumab and chemotherapy. Addition of pertuzumab to trastuzumab as adjuvant therapy resulted in a statistically significant increase of 3-year disease-free survival [10], while Hurvitz et al. recently reported rates of pathological complete response as high as 55\% after neoadjuvant dual HER2 blockade, in conjunction with chemotherapy [11]. Moreover, extended adjuvant treatment with the tyrosine kinase inhibitor neratinib maleate after trastuzumab-based therapy significantly decreased invasive disease-free survival events [8]. Furthermore, palbociclib, ribociclib and abemaciclib have been approved for the treatment of advanced or metastatic HER2-negative/ER-positive breast cancer [1].

Considering both clinicopathologic and genetic patient characteristics, an important step toward personalized breast cancer treatment has been achieved. Integrating age, Tumor, Node, Metastasis (TNM) staging and grade, as well as ER/PR and HER2 status into primary decision-making on systemic therapy has substantially reduced recurrence and cancer-related death rates. Expanding the list of available targeted drugs, including pertuzumab and neratinib maleate, has improved the efficacy of personalized neoadjuvant or adjuvant treatment.

Despite all these advances, a substantial proportion of HER2-positive patients face high risk of relapse and death, depending on TNM stage. Moreover, moderate to slow progress has been observed for triple-negative breast cancer, with chemotherapy remaining the standard treatment, in the absence of biomarker-directed effective therapy. The lack of robust predictive biomarkers for accurate personalized primary and secondary decision-making, coupled with the shortage of multiple effective drugs to overcome established extensive genetic heterogeneity [5], explain the persisting relapse and death rates. One of the most rational and promising perspectives currently lies in genome analysis for the development of both biomarkers and drugs.

\section{Genome analysis: translational progress \& challenges}

The public concern surrounding breast cancer, owing to high numbers of women at risk, have led to significant efforts at early, noninvasive diagnosis through liquid biopsies, combining targeted next-generation sequencing (NGS) of plasma cell-free DNA (cfDNA) and protein biomarkers, aiming to complement current screening measures. However, despite the innovative concept of a blood-based test for early detection, the largest and most well-designed study by Cohen et al. [12] has reported a quite low sensitivity of 33\% in the diagnosis of early breast cancer, the lowest for any of the eight cancer types examined. Thus, considering the already effective screening strategy against breast cancer, as well as the doubtful accuracy of cfDNA-NGS in early-stage tumors [13], further refinements are needed before NGS-based liquid biopsies could be implemented into clinical practice, especially for breast cancer.

Moreover, the high incidence and commercial interest in breast cancer have resulted in several genomic studies of considerable size, allowing for the valid identification of cancer genes and the delineation of the underlying mutational landscape. A landmark study by Lawrence et al. [5] on 21 tumor types, which introduced the significance of validity in novel genomic discoveries and proposed specific guidelines for achieving it, applied whole-exome sequencing (WES) on 4742 tumor samples, with 892 breast cancers among them. The researchers identified 33 novel cancer driver genes, with one of them identified in breast tumors. Additional novel cancer genes have been detected by other large-scale reports, including a WES study reporting a novel susceptibility gene [14], as well as the largest whole-genome sequencing (WGS) study by Nik-Zainal and colleagues on 560 patient-derived samples [15]. The latter identified five novel cancer driver genes, while activated fusion genes and noncoding driver mutations were rarely found.

Beyond static, single biopsy-based genomic studies, the recent advent of integrated NGS systems has enabled the application of innovative, breakthrough designs for the exploration of intratumor heterogeneity and tumor evolution in time and space. This comprehensive molecular characterization aims to clarify the origins of therapeutic resistance and relapse through multiregional NGS and single-cell genome sequencing. An outstanding example is a study by Yates et al. [16] which applied NGS on 303 multiregional tumor samples from 50 patients, with obtained specimens before and after neo-adjuvant treatment (NAT) for 18 patients. The authors identified variable levels of intratumor heterogeneity with 13 of 50 patients carrying subclonal druggable mutations. Moreover, subclonal diversification in response to NAT was observed in five of 18 patients, with specific alterations being identified as 
drivers of chemotherapy resistance. Dynamic spatiotemporal clonal tumor evolution is hereby strongly suggested, in contrast to smaller studies supporting the pre-existence and temporal stability of an aggressive cell subpopulation within the primary tumor, driven by chromosomal rearrangements [17].

On this basis and given the identification of intratumor heterogeneity even at the single-cell level, a breakthrough technique for sequencing individual cancer cells has recently emerged. Nevertheless, single-cell sequencing has not yet resolved the aforementioned dispute with controversial and inconclusive results being reported. On one hand, Baslan and colleagues [18] have reported dynamic clonal diversification after analyzing 332 single cells from two ER-positive breast cancers, supporting the Darwinian model of evolution. Wang et al. [19] have attributed this evolving subclonality to point mutational divergence, while they found chromosomal rearrangements to be early evolutionary events which remained stable over time. By contrast, one of the largest single-cell sequencing analyses on breast cancer identified minor, nonclonal cell subpopulations with high metastatic capacity, concluding that early emergence and stability of copy number alterations could be a driving force of cancerous invasion, metastasis and therapeutic resistance [20]. This study supports a new model of punctuated clonal evolution, as opposed to gradual diversification, earlier described by Navin $e$ al. on two breast cancers [21].

Although single-biopsy NGS analysis, including WES, WGS and RNA sequencing, can identify breast cancer risk genes, valid discovery of novel cancer driver genes involved in familial and sporadic cancer requires large-scale studies, according to recent recommendations [5], with only a handful meeting these criteria. Indeed, based on the polygenic model of breast cancer risk, further genomic research, including the identification of noncoding variants in susceptibility genes [22], is required for appropriate genetic screening among women with familial predisposition to cancer. However, in the therapeutic setting and considering the dynamic evolution of genomic clones in time and space [23], conventional static single-biopsy NGS analysis is unsuitable to develop prognostic and predictive biomarkers essential for overcoming intrinsic and acquired therapeutic resistance [24,25].

\section{Conclusion \& future perspective}

Despite advances in breast cancer risk management for prevention and treatment, personalized primary decisionmaking remains a researcher's dream. Beyond $B R C A 1 / 2$ genes, much of the genetic contribution to breast cancer risk remains unknown [26]. Similarly, in spite of advancements toward individualization of therapy based on clinicopathologic and molecular patient characteristics, neither robust biomarkers to precisely predict intrinsic and acquired therapeutic resistance, nor an expanded list of targeted drugs to effectively target the comprehensive intrapatient mutational landscape have yet been developed. The innovative identification of intratumor heterogeneity and circulating genomic subclones as biomarkers for tailored treatment is highly promising, but still, validation is required. The vast majority of available genomic studies is limited by small sample size, high heterogeneity of baseline clinical and TNM-staging data and the lack of a strict protocol for accurate detection of spatiotemporally evolving, 'resistant' genomic subclones. Further progress and refinement of integrated NGS systems, such as cfDNA-NGS and single-cell genome and/or RNA sequencing, could enable crucial translational implications.

In summary, the recent expansion of the targeted drug list has further improved patient survival. However, patients with stage III or locally advanced disease, as well as triple-negative breast cancer, remain at high risk for therapeutic resistance, relapse and death. Rational, translational research with focus on static and dynamic genome analysis has already returned exciting data on the potential development of prognostic and predictive biomarkers, as well as new targeted drugs, through the discovery of novel oncotargets. Although these data shape the innovative roadmap toward precise individualization of breast cancer therapy, an evidence-based strategy is essential to validate promising breast cancer genome analysis in time and space.

\section{Financial \& competing interests disclosure}

The authors have no relevant affiliations or financial involvement with any organization or entity with a financial interest in or financial conflict with the subject matter or materials discussed in the manuscript. This includes employment, consultancies, honoraria, stock ownership or options, expert testimony, grants or patents received or pending, or royalties.

No writing assistance was utilized in the production of this manuscript.

\section{References}

1. National Comprehensive Cancer Network, Inc. (2018). www.nccn.org/

2. Siegel RL, Miller KD, Jemal A. Cancer statistics, 2018. CA Cancer J. Clin. 68(1), 7-30 (2018).

3. Zardavas D, Irrthum A, Swanton C, Piccart M. Clinical management of breast cancer heterogeneity. Clin. Oncol. 12(7), 381-394 (2015). 
4. Narod SA. BRCA mutations in the management of breast cancer: the state of the art. Nat. Rev. Clin. Oncol. 7(12), 702-707 (2010).

5. Lawrence MS, Stojanov $\mathrm{P}$, Mermel $\mathrm{CH}$ et al. Discovery and saturation analysis of cancer genes across 21 tumour types. Nature 505(7484), 495-501 (2014).

6. Nair A, Chung HC, Sun T et al. Combinatorial inhibition of PTPN12-regulated receptors leads to a broadly effective therapeutic strategy in triple-negative breast cancer. Nat. Med. 24(4), 505-511 (2018).

7. Hyman DM, Piha-Paul SA, Won H et al. HER kinase inhibition in patients with HER2- and HER3-mutant cancers. Nature 554(7691), 189-194 (2018).

8. Martin M, Holmes FA, Ejlertsen B et al. Neratinib after trastuzumab-based adjuvant therapy in HER2-positive breast cancer (ExteNET): 5-year analysis of a randomised, double-blind, placebo-controlled, Phase 3 trial. Lancet Oncol. 18(12), 1688-1700 (2017).

9. Gianni L, Pienkowski T, Im YH et al. 5-year analysis of neoadjuvant pertuzumab and trastuzumab in patients with locally advanced, inflammatory, or early-stage HER2-positive breast cancer (NeoSphere): a multicentre, open-label, Phase 2 randomised trial. Lancet Oncol. 17(6), 791-800 (2016).

10. Von Minckwitz G, Procter M, De Azambuja E et al. Adjuvant pertuzumab and trastuzumab in early HER2-positive breast cancer. $N$. Engl. J. Med. 377(2), 122-131 (2017).

11. Hurvitz SA, Martin M, Symmans WF et al. Neoadjuvant trastuzumab, pertuzumab, and chemotherapy versus trastuzumab emtansine plus pertuzumab in patients with HER2-positive breast cancer (KRISTINE): a randomised, open-label, multicentre, Phase 3 trial. Lancet Oncol. 19(1), 115-126 (2018).

12. Cohen JD, Li L, Wang Y et al. Detection and localization of surgically resectable cancers with a multi-analyte blood test. Science 359(6378), 926-930 (2018).

13. Cohen JD, Javed AA, Thoburn C et al. Combined circulating tumor DNA and protein biomarker-based liquid biopsy for the earlier detection of pancreatic cancers. Proc. Natl Acad. Sci. USA 114(38), 10202-10207 (2017).

14. Cybulski C, Carrot-Zhang J, Kluzniak W et al. Germline RECQL mutations are associated with breast cancer susceptibility. Nat. Genet. 47(6), 643-646 (2015).

15. Nik-Zainal S, Davies H, Staaf J et al. Landscape of somatic mutations in 560 breast cancer whole-genome sequences. Nature 534(7605), 47-54 (2016).

16. Yates LR, Gerstung M, Knappskog S et al. Subclonal diversification of primary breast cancer revealed by multiregion sequencing. Nat. Med. 21(7), 751-759 (2015).

17. Tang $\mathrm{MH}$, Dahlgren $\mathrm{M}$, Brueffer $\mathrm{C}$ et al. Remarkable similarities of chromosomal rearrangements between primary human breast cancers and matched distant metastases as revealed by whole-genome sequencing. Oncotarget 6(35), 37169-37184 (2015).

18. Baslan T, Kendall J, Ward B et al. Optimizing sparse sequencing of single cells for highly multiplex copy number profiling. Genome Res. 25(5), 714-724 (2015).

19. Wang Y, Waters J, Leung ML et al. Clonal evolution in breast cancer revealed by single nucleus genome sequencing. Nature 512(7513), 155-160 (2014).

20. Gao R, Davis A, McDonald TO et al. Punctuated copy number evolution and clonal stasis in triple-negative breast cancer. Nat. Genet. 48(10), 1119-1130 (2016).

21. Navin N, Kendall J, Troge J et al. Tumour evolution inferred by single-cell sequencing. Nature 472(7341), 90-94 (2011).

22. Rheinbay E, Parasuraman P, Grimsby J et al. Recurrent and functional regulatory mutations in breast cancer. Nature 547(7661), 55-60 (2017).

23. Eirew P, Steif A, Khattra J et al. Dynamics of genomic clones in breast cancer patient xenografts at single-cell resolution. Nature 518(7539), 422-426 (2015).

24. Roukos DH. Spatiotemporal diversification of intrapatient genomic clones and early drug development concepts realize the roadmap of precision cancer medicine. Drug Discov. Today 22(8), 1148-1164 (2017).

25. Murtaza M, Dawson SJ, Tsui DW et al. Non-invasive analysis of acquired resistance to cancer therapy by sequencing of plasma DNA. Nature 497(7447), 108-112 (2013).

26. Michailidou K, Lindstrom S, Dennis J et al. Association analysis identifies 65 new breast cancer risk loci. Nature 551(7678), 92-94 (2017). 